\title{
Large Displacement Flexible Micro Actuators
}

\author{
P. Dhara Mallesh ${ }^{1}$, \\ ${ }^{I}$ Department of Mechanical Enginnering, Indian Institute of Technology, Bombay, India
}

\begin{abstract}
This paper discusses a major application of flexible micro actuators in Micro Air Vehicles (MAV). Design, Kinematics, flapping patterns, performance tests of flapping wing mechanism which replicates dragonfly and corresponding results are discussed in this paper. This paper also discusses artificial foldable wing models by mimicking the unfolding/folding of beetle hind wing in which the actuation is triggered by electrically activated Shape Memory Alloy (SMA) wires.
\end{abstract}

Keywords: Micro Air Vehicles, Modified slider-crank (MSC), Flapping patterns, Hind wing, Shape Memory Alloy

\subsection{Micro-air-vehicles (MAVs)}

\section{Introduction}

Which are belongs to a class of Unmanned aerial vehicles, characterized by small vehicle size $(<10 \mathrm{~cm})$ and low flight speed $(<10 \mathrm{~m} / \mathrm{s})$. The need for these small aero vehicles is largely for aerospace engineering applications equipped with video cameras, transmitters, or sensors, these miniaturized aerial vehicles can perform surveillance, reconnaissance, targeting, or biochemical sensing tasks at $\mathrm{D}^{3}$ environments (Dull, Dirty, Dangerous).

Mav's are three types

i. $\quad$ Fixed wing MAVs.

ii. Rotary wing MAVs.

iii. Flapping wing MAVs.

i. Fixed wing MAVs:

These types of Wing MAVs require higher, forward flight speeds to stay airborne so these are useful to cover longer distances .But these are unable to effectively maneuver inside structures such as buildings. So Fixed-wing MAVs do not have the required agility for obstacle-avoidance in indoor flight, and are incapable of hovering.

\section{ii. Rotary wing MAVs:}

These types of wing MAVs allow craft to hover and move in any direction. Even though rotary- wing MAVs are capable of vertical-take-off-and-landing capability, they too noisy.

iii. Flapping wing (Flexible wing) MAVs:

Flapping wing MAVs are superior to both fixed wing and rotary wing types due to significant aerodynamic benefits. Over a million dissimilar species of insects fly with flapping wings to produce lift and thrust, and more than 10,000 different kinds of birds and bats flap their wings for locomotion.

As the elevated flight speed increases, the aerodynamic forces acting on the wings would become larger and larger. the significant aerodynamic forces acting on flexible membrane airfoils/wings would cause chord wise profile changes to adapt the incoming flows automatically (i.e., the chord profiles of the flexible membrane wings would be adjusted automatically) to balance the pressure differences between the upper and lower surfaces of the flexible membrane airfoils/wings. When the aerodynamic forces acting on flexible membrane airfoils/wings become more significant, the trailing edges of the flexible membrane airfoils/wings would be deflected and lifted up from its original designed position, which would reduce the effective angle of attack of the flexible membrane airfoils/wings with respect to incoming flow [1]
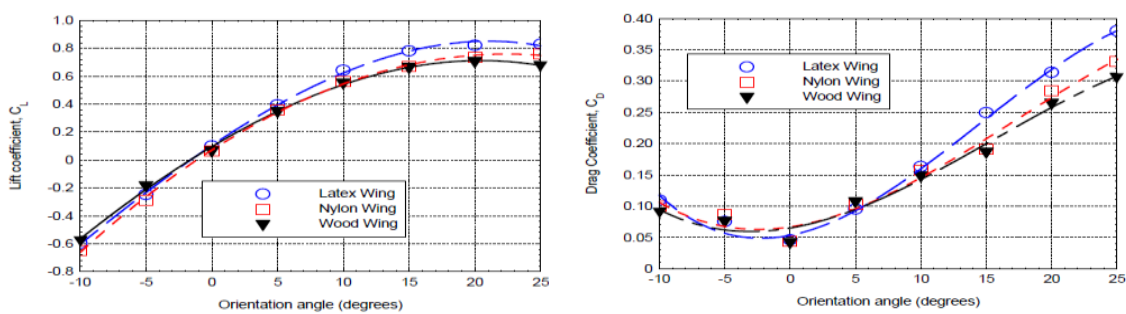

Fig.1: Variation of lift and drag coefficients for latex, nylon, wood wings [1] 


\subsection{Flapping wing v/s Rigid wing}

- Fixed wing aircraft get their primary lift generation as function of forward airspeed and amount of wing span \& surface area. For MAV flying in confined spaces very slow speeds are needed to avoid running into obstacles.

- Flapping wings get their lift not from forward airspeed of the aircraft but from flapping motion of the wing. Because of this there is less reliance on forward airspeed to generate lift and the amount of lift (and thrust) generated can be controlled by how fast the wings beat. This conceptually solves the issue for a fixed wing MAV trying to fly at very slow airspeeds or even hover.

\subsection{Shape Memory Alloy}

A shape-memory alloy is an alloy that "remembers" its original, cold-forged shape returning the predeformed shape by heating. This material is a lightweight, solid-state alternative to conventional actuators such as hydraulic, pneumatic, and motor-based systems. Shape-memory alloys have applications in industries including medical and aerospace. So Shape Memory Alloys (SMA's) are novel materials which have the capability to return to a predetermined shape when heated. When an SMA is cold, or below its transformation temperature, it has a very low yield strength and can be deformed quite easily into any new shape which it will retain. However, when the material is heated above its transformation temperature it undergoes a change in crystal structure which causes it to return to its original shape. If the SMA encounters any resistance during this transformation, it can generate extremely large forces. This phenomenon provides a unique mechanism for remote actuation.

The SMA becomes stiffer and the crystal structure transforms to austenite when heated within the transformation temperature range. Above the transformation temperature range, the crystal structure is purely austenite (Figure 2).

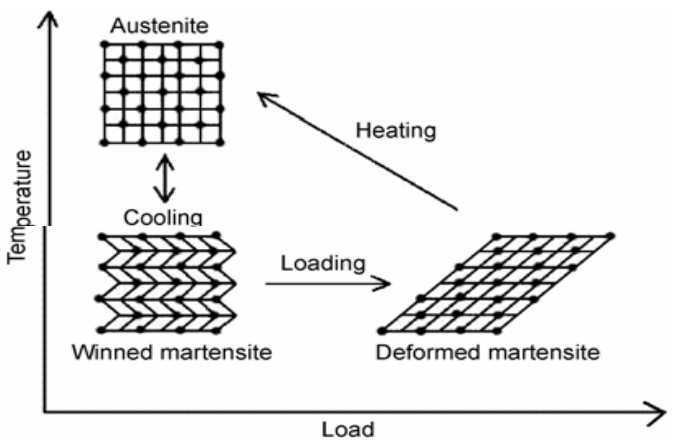

Fig.2: SMA phase transformation [5]

\section{Design Of A Flapping Wing Mechanism And A Micro Aerial Vehicle Using A Rotary Actuator}

In January 2010, the Tamkang University(TKU) in Taiwan realized autonomous control of the flight altitude of an 8-gram, 20-centimeter wide, flapping-wing MAV. In 2008, the TU Delft University in the Netherlands developed the smallest ornithopter fitted with a camera, the Delfly Micro[2]. In the present circumstances where security is the main issue for all, the popularity of MAVs is increasing in military, surveillance, planetary exploration, search-and-rescue and many other applications. As discussed early the importance and advantages with flapping wings the scientific attention has been turned on to the applications of flapping wings, the motions of which are based on those of birds and insects. If low Reynolds Number aerodynamics is exploited, the flapping wing MAVs can be more efficient than the MAVs of conventional design [3]. In addition, multi-mobility of the flapping wing MAVs, which introduce forward flapping flight, hovering and gliding as well as crawling on the ground and water surface, is another advantage over the other MAVs that require a new actuator every time a new motion is introduced.

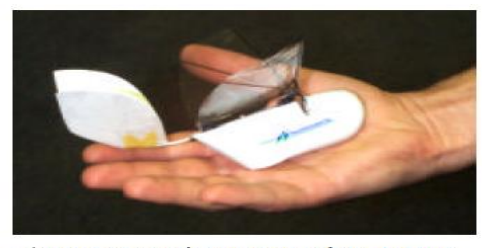

a) super capacitor-powered prototype

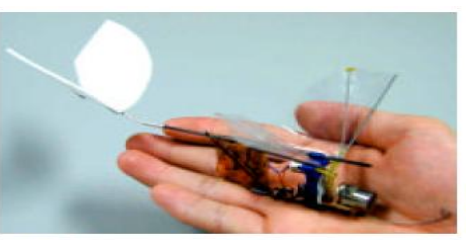

b) battery-powered prototype

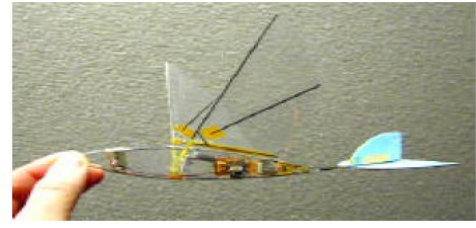

c) battery-powered prototype with a radio control system

Microbat Prototypes

Fig.3: The first palm sized electric ornithopter that successfully flew forwards. [4] 
This microbat used aero-elastic effects of wings for forward propulsion. After this so many MAVs are invented which uses the flapping patterns of nature's best flying and hovering creatures such as such as dragonflies, hummingbirds and bees consist of a flap or stroke and rotation or twisting of the wing. So the analysis of flapping wing motions of such creatures is important as they give clues to design better flying machines at smaller scales in addition to increased efficiency.

Here in this paper the design of a flapping wing mechanism that replicates flapping patterns of dragonflies is discussed. To generate four different flapping patterns here a modified slider-crank (MSC) mechanism is used. In this mechanism a rotary actuator is used to get the motion and generates four distinct flapping patterns.

\subsubsection{Flapping patterns}

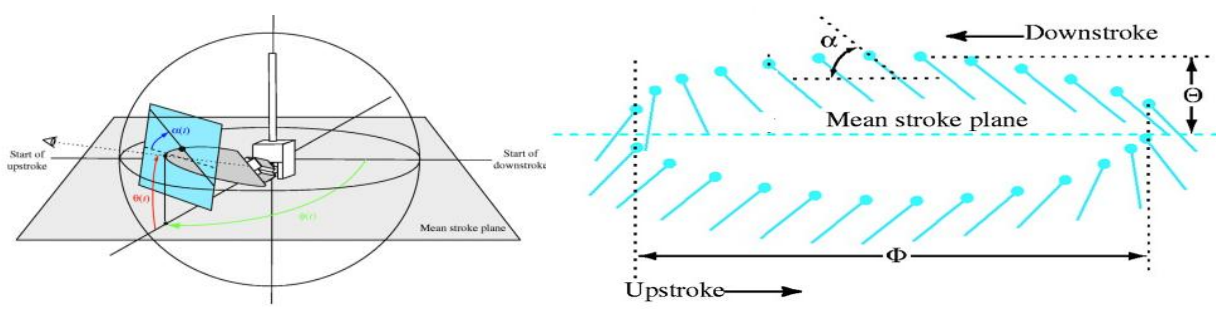

Fig.4: kinematic representation of a flapping wing. [4]

Fig.4 shows the three Euler angles that define the wing position at each instant in time. The mean stroke plane is a horizontal slice through the sphere described by the radial coordinates of the wing tip. Instantaneous stroke position, $\varphi(t)$, is defined as the angular position of the wing in the mean stroke plane, measured from start of down stroke to start of upstroke. Instantaneous stroke deviation, $\theta(t)$, is defined as the angle that the base-to-tip line on the wing makes with the mean stroke plane.

A plane that is normal to the base-to-tip line of the wing (shown in blue) cuts through the wing at the wing chord, shown here as a line with a filled circle denoting the leading edge. The instantaneous angle of attack, $\alpha(t)$, is the angle that the wing chord makes with the tangent of the wing's trajectory. Flight patterns of flying creatures are shown in FIG-5 which compares the flight patterns of flying creatures including the dragonflies, hovering insects and hummingbirds. Shown in FIG. 4A is a flight pattern of hovering insects and hummingbirds, which use normal hovering FIG. 4B, meanwhile, illustrates the flight pattern of dragonflies and true hoverflies, Hovering along an inclined stroke plane is advantageous in comparison to normal hovering since (1) power is required for one half stroke only and (2) aerodynamic drag can be used to support weight.
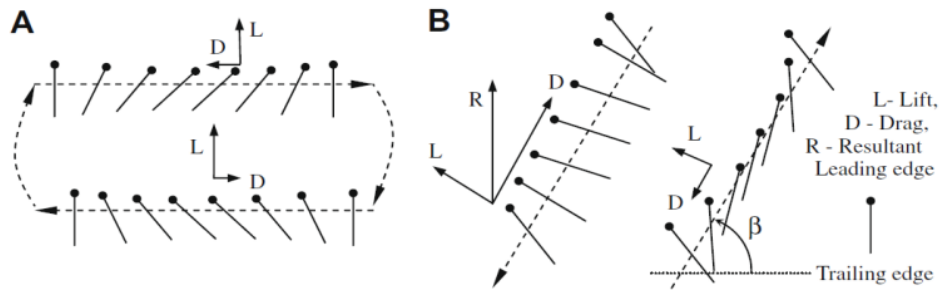

Fig.5: Hovering patterns of dragon flies, A: Normal hovering, B: hovering using inclined stroke plane. [4]

\subsubsection{Mechanism}

Here in this paper Modified Slider Crank (MSC) mechanism is discussed [4]. By taking flying nature and characteristics from flying creatures the MSC mechanism was designed such that efficient flapping patterns can be replicated. Fig. 6 shows the MSC mechanism that is capable of generating four distinct flapping patterns using a single rotary actuator where Fig. 6A and B illustrates a design and its kinematic structure respectively. The mechanism consists of a crank, coupler, drive-arm, pivot and main-arm spaced by specific distances. The coupler's design is crucial and consists of four rotational joints. The crank and the coupler form a rotational joint, the coupler and the drive-arm form a rotational joint. The crank, drive-arm and main-arm axes lie in the same plane: the stroke plane. As the crank rotates it pushes or pulls the drive-arm along an arc (since the drivearm is constrained by a pivot) creating wing strokes. The crank's rotation also inclines the coupler with respect to the stroke plane. This inclination of the coupler twists or rotates the drive-arm and the wing. Hence wing flapping with rotation is achieved through the rotation of a crank. The MSC mechanism was inspired from a slider-crank mechanism, but differs by the facts that (1) the slider (coupler-drive arm joint) in the MSC mechanism moves along an arc as it is constrained by the pivot and (2) the coupler-drive-arm joint in 
the MSC is formed by a revolute joint whose axis is orthogonal to the drive-arm and coupler axis. In a normal slider-crank mechanism the coupler-slider joint is a rotational joint whose axis, if not in the same plane, is parallel to the crank axis. Hence the inclination of the coupler link in a normal slider-crank mechanism is nulled unlike in the MSC mechanism.

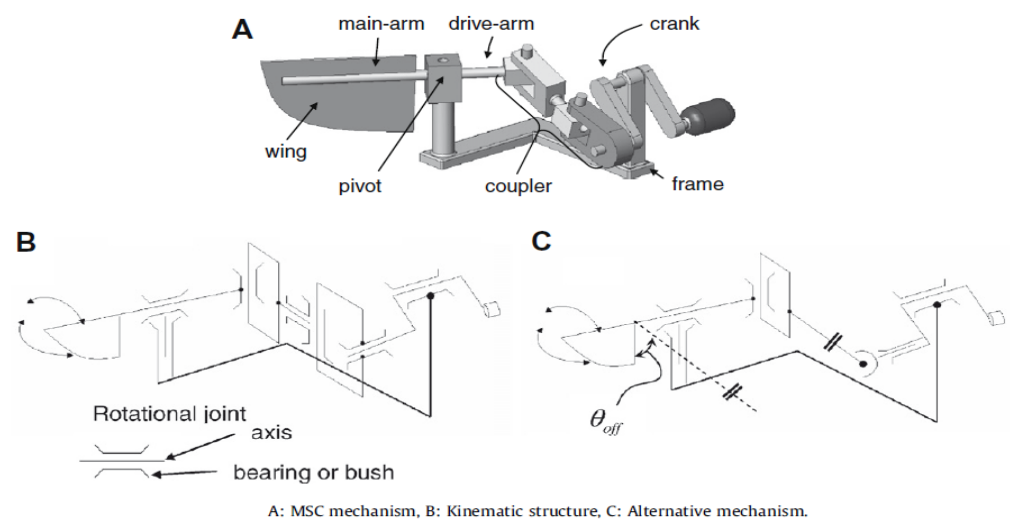

Fig. 6: Modified Slider Crank Mechanism (MSC) [4]

But prototype should have less weight so reducing the number of components is very important aspect in the design of this mechanism. Fig-6 C shows an alternative mechanism where the two joints of the coupler are replaced by a spherical joint. Here all the dimensions of the components are small like the crank length is 3$5 \mathrm{~mm}$. At this small size it is difficult to construct a rotational or a spherical joint along length of the coupler. Hence flexible materials such as plastic and thin steel plates were used to build the coupler to allow for slight torsion and bending along the length of the coupler.

\subsubsection{Kinematics and flapping patterns}

Fig. 7 shows the notations necessary to describe the kinematics of the alternative MSC mechanism shown in Fig. 6C. The MSC mechanism is designed such that the transmission angle between two joints is symmetric during both strokes. Given the length of the crank $\left(r_{c}\right)$, desired maximum coupler inclination $\left(\theta_{c}\right)$ and maximum angle of swing or flapping $\left(\theta_{s}\right)$ that the mechanism has to generate, the dimension of each component and its spacing can be calculated. When the link $\mathrm{OZ}^{\prime}$ is oriented by $\theta_{r}=90^{\circ}$, the length $\mathrm{CZ}^{\prime}$ can be calculated in terms of the length of the crank $r_{c}$ and the desired maximum coupler inclination $\theta_{c}$ as

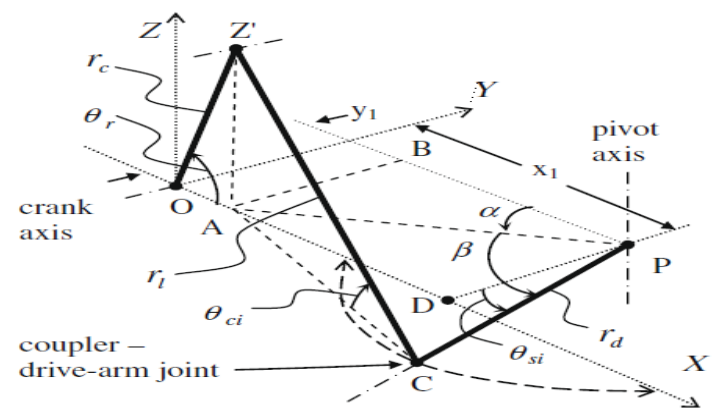

$r_{l}=r_{c} / \sin \left(\theta_{c}\right)$

Fig.7: Kinematic analysis of the mechanism. [4]

When $\theta_{r}=0^{\circ}$ and $180^{\circ}$, the coupler-drive-arm joint will lie on the XY plane at $Y=0, X=r_{c}+r_{l}$ and $X=r_{l}-r_{c}$ respectively. The value of $\theta_{s i}$ ranges between $\pm \theta_{s} / 2$. Hence the drive-arm length is given as, $r_{d}=r_{c} / \sin \left(\theta_{s} / 2\right)$

As a result, the pivot distance $\left[\mathrm{x}_{1}, \mathrm{y}_{1}\right]$ is given as,

$X_{l}=\left(r_{c}+r_{l}\right)-\left(r_{d} \sin \left(\theta_{s} / 2\right)\right)$

$Y_{l}=r_{d} \cos \left(\theta_{s} / 2\right)$

Given the instantaneous position of the crank $\left[r_{c}, \theta_{r}\right]$ the position of the drive-arm $\left[r_{d}, \theta_{s i}\right]$ can be calculated from triangle ABP:

$O A=r_{c} \cos \left(\theta_{r}\right)$

$\alpha=\cos ^{-1}(B P / A P)$

$\theta_{c i}=\sin ^{-1}\left(A Z^{l} / r_{l}\right)$

$A C=r_{l} \cos \left(\theta_{c i}\right)$ 
$\beta=a \cos \left[\left(r_{d}^{2}+A P^{2}-A C^{2}\right) /\left(2 r_{d} A P\right)\right]$

$\theta_{s i}=\alpha+\beta$

where $\theta_{c i}$ and $\theta_{s i}$ are the instantaneous angles of the coupler and drive-arm respectively. The crank axis and the drive-arm axis lie in the same plane, the stroke plane. If either axis is moved along the $Z$ axis the timing between each stroke can be adjusted and asymmetry can be achieved.

\subsubsection{Prototype MAV}

The prototype was fitted with an asymmetric pair of tapered wings (span of $9 \mathrm{~cm}$ and inner rib of $6 \mathrm{~cm}$ ) and tested on a simple balance. The prototype was found to hover using flapping pattern 3 with an external power supply of $3.4 \mathrm{~V}$ at $190 \mathrm{~mA}, \mathrm{f}=8.8 \mathrm{~Hz}$. A significant load of $0.92 \mathrm{~g}$ (a Lithium Polymer battery) was attached to the body of the prototype and tested for hovering capability. The prototype continues to exhibit hovering with an increased external input of $4.4 \mathrm{~V}$ at $220 \mathrm{~mA}, \mathrm{f}=11.5 \mathrm{~Hz}$.

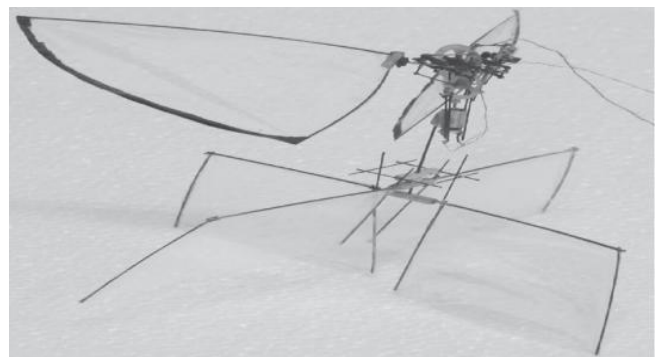

Final flapping wing prototype MAV [4].

This prototype generates four distinct flapping patterns using a DC motor. The above figure shows the two winged MAV prototype incorporating twin MSC mechanisms that exhibits constrained hovering and forward flight, weighs $3.35 \mathrm{~g}$ (3.2 g excluding the four flaps) and measures $200 \mathrm{~mm} \_200 \mathrm{~mm} \_150 \mathrm{~mm}$.

\subsection{Artificial Foldable Wing Models by Mimicking the Unfolding /Folding Mechanism of a Beetle Hind Wing}

This part presents an experimental research [5] aiming to understand a synthetic hind wing that can imitate the wing unfolding motion of Allomyrina dichotoma, an insect in coleopteran order. Based on the understanding of working principles of beetle wing folding/unfolding mechanisms, the hind wing unfolding motion is mimicked by using an artificial muscle actuator. In this work, two types of artificial wings are demonstrated successfully that they can be unfolded by actuation of shape memory alloy wires to provide actuation force at the wing base and along the leading edge vein.

Here it's also presented the improvements to foldable artificial hind wing which is mentioned above. Multiple hinges, which were implemented to mimic the bending zone of a beetle hind wing, were made of small composite hinge plates and tiny aluminum rivets. Folding and unfolding actions were triggered by electricallyactivated Shape Memory Alloy (SMA) wires. For wing folding, the actuation characteristics of the SMA wire actuator were modified through heat treatment.

For a long time service the wings of MAVs may easily broken by external loading so in the reference [5] a beetle, Allomyrina dichotoma (Figure 8) is taken as a reference to mimic the folding mechanism as this particular insect has a foldable wing mechanism. The beetle folds and stows hind wings beneath fore wings (Figure 9) for ground locomotion and unfolds them only for flight, which enables a long term operation. In addition, Allomyrina Dichotoma is one of the largest flying insects, and its flight principle is relatively easy to observe with bare eyes. Here they [5] proposed two types of artificial foldable wing models, proving that they can be unfolded by actuation of shape memory alloy (SMA) wires installed along the leading edge veins and at the wing base.

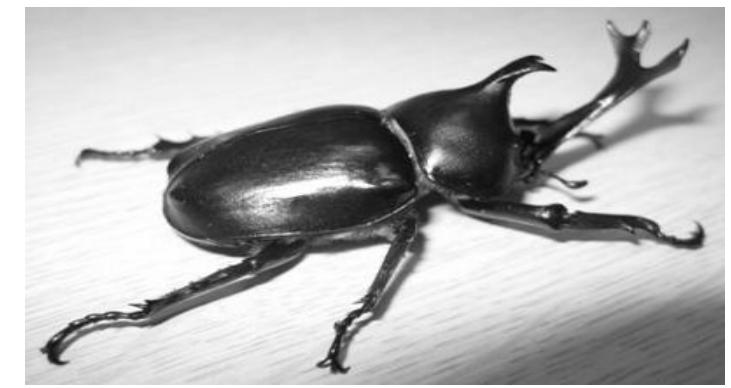



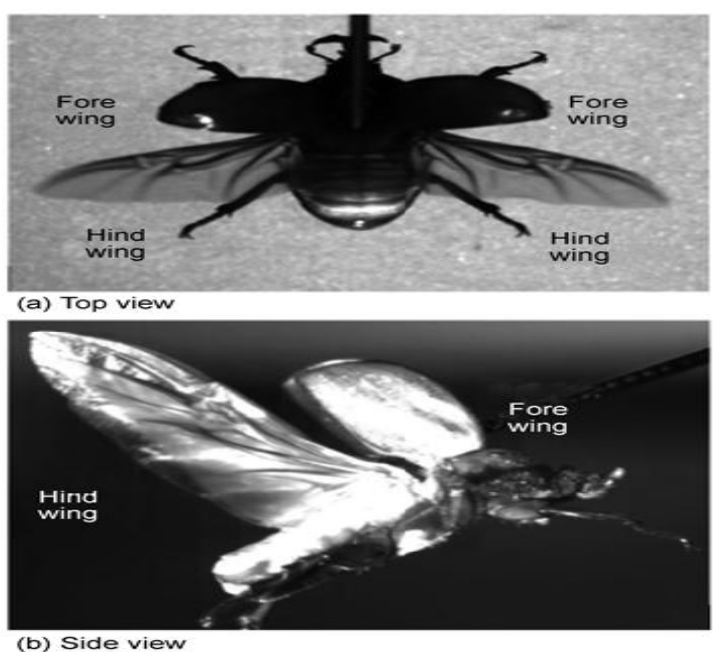

Fig. 9: Fore wings (elytra) and hind wings. [5]

\subsubsection{Wing folding/unfolding mechanism}

The wings of this beetle have two parts, a pair of fore wings called elytra and a pair of hind wings (Figures 9(a) and (b)). Elytra are rigid wings acting as covers of the hind wings during ground locomotion. Thus, hind wings are folded and stowed under the elytra during crawling. A deformable hind wing comprises stiff veins and membranous part as shown in Figure 10. When unfolding wings, a beetle opens elytra first and then releases the hind wings.

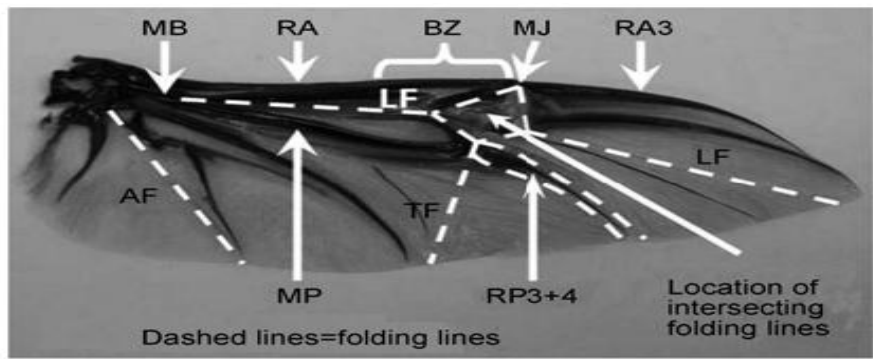

Fig.10: A hind wing of Allomyrina dichotoma. [5]

\subsubsection{Out-of-plane wing folding/unfolding}

This mechanism have four stiff panels, four folding lines, and a point of intersection (Figure 11), where those folding lines are borders and also hinges of the four stiff panels that meet each other at a point of intersection. A wing is unfolded along the four folding lines by increasing the angle $\varepsilon$ between its base panels as shown in Figure 11(a). Inversely, as shown in Figure 11(b), the folding configuration can be achieved by reducing the angle $\varepsilon$.

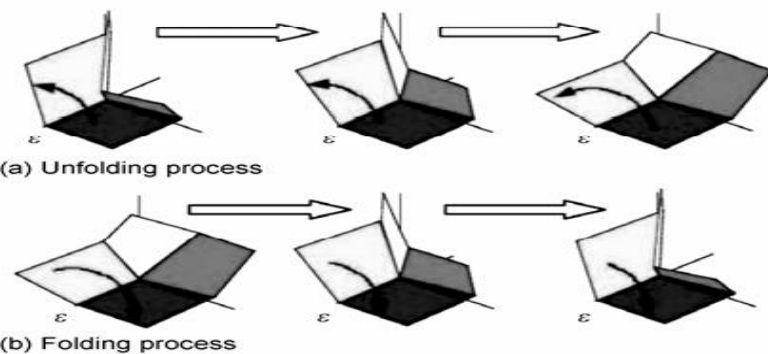

Fig.11:Basic folding and unfolding mechanisms [5]

\subsubsection{In-plane wing folding/unfolding}

The basic folding/unfolding mechanism explained by Haas and Wootton[7] involves a large out-ofplane motion during wing opening process. However, from [5] it is clear that the forced folding/unfolding process based on images captured by a digital camera recorder, it is found that the unfolding process of hind wings of Allomyrina dichotoma occurs nearly in a plane. 


\subsubsection{Design of artificial foldable wings}

Here the basic design of artificial foldable wing for both out of plane and in plane wings based on folding/unfolding principles and also the improvements in this design.

Both of them are designed as alike as possible to the real one in terms of dimensions, vein thickness, membrane thickness, wing contour, and vein pattern. Here for the out-of-plane unfolding model, the thickness of vein and membrane are not taken as of a real beetle wing, thus the four wing panels are flat and have the same thickness. A small panel is added at the inner side of wing articulation for the SMA wire installation as shown in Figure 12. This model has a span length of $7.5 \mathrm{~cm}$ excluding the added panel, which is typically 1.5 times longer than that of a real hind wing.

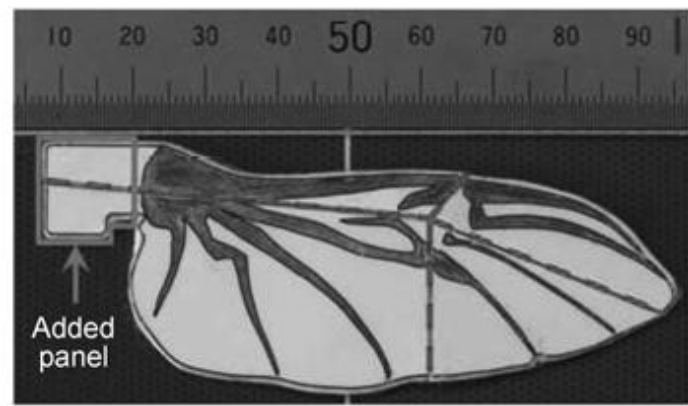

Fig. 12: The out-of-plane wing model [5]

Figure 13 shows an artificial foldable hind wing with $10.5 \mathrm{~cm}$ of span length, which is typically two times longer than that of a real hind wing of Allomyrina Dichotoma species. Membrane of this model is made of $15 \mu \mathrm{m}$ thick polypropylene film. Meanwhile, the vein structure is made of $0.4 \mathrm{~mm}$ thick glass/epoxy composites. This composition is chosen because it is lightweight and strong enough. In order to acquire accurate dimension, each part of this model is designed by using CAD software and the cutting process of composite material is done by a laser cutting machine.

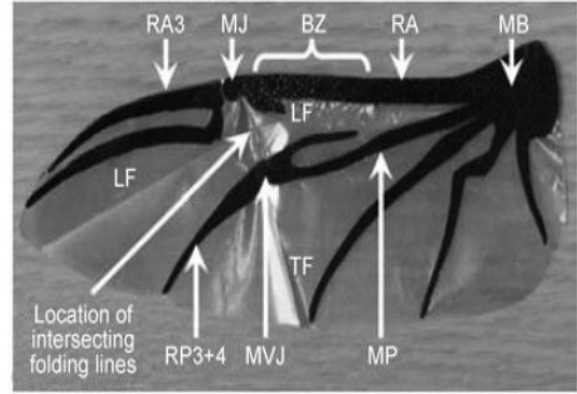

Fig.13: The in-plane wing model [5]

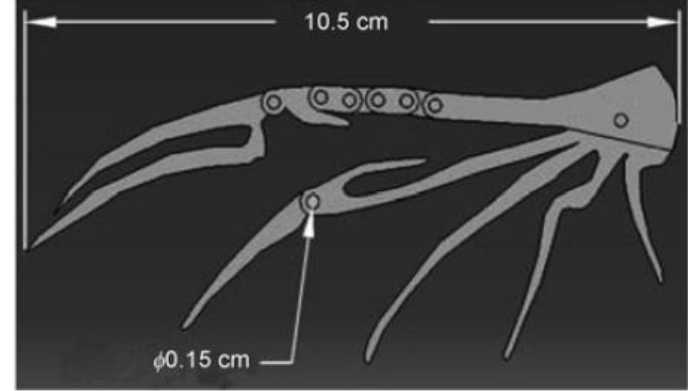

(CAD design of vein structure of the in-plane wing model)

As shown in the above figures the diameters of the holes are all $1.5 \mathrm{~mm}$. The shaft is made of $1.5 \mathrm{~mm}$ diameter carbon rod and the size of each hole slightly smaller to construct interference fit hinge, so that the shape of the wing structure can be kept straight after assembly.

\subsubsection{Improved model}

Initially multiple in-plane hinges were used to mimic the $\mathrm{BZ}$ of a real beetle hind wing, as shown in FIG-14 a piece of composite hinge plate and two short carbon rods were used to form each in-plane hinge. As shown in FIG 14a, each carbon rod was tightly fit (interference fit) to a hole in its own hinge plate because the free ends of the rods could not be flared. Because of this, friction in the in-plane hinges was relatively large, and fast wing folding and unfolding motions were impossible. In the current work, the carbon rod is replaced with a tiny rivet to reduce friction in the in-plane hinges, as shown in FIG 14b. In the current artificial wing, the diameter of each hole was slightly larger than $1.15 \mathrm{~mm}$ and the shaft diameter of an aluminum rivet was 1.15 $\mathrm{mm}$. The rivet buck-tails were flared to obtain tight clearance in each in-plane hinge. Thus, a sliding fit was available. In this way, the friction in each hinge can be reduced and maintain a straight wing shape after assembly. Also, the artificial wing did not arbitrarily fluctuate during flapping motion. 


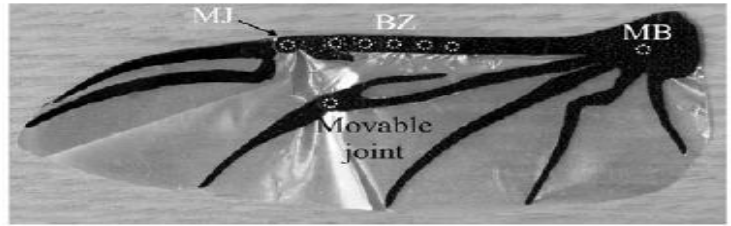

(a) Previous mode1

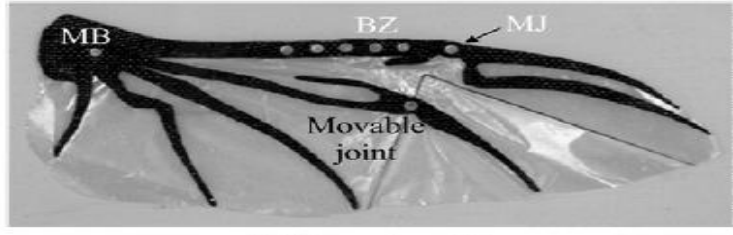

(b) Current model

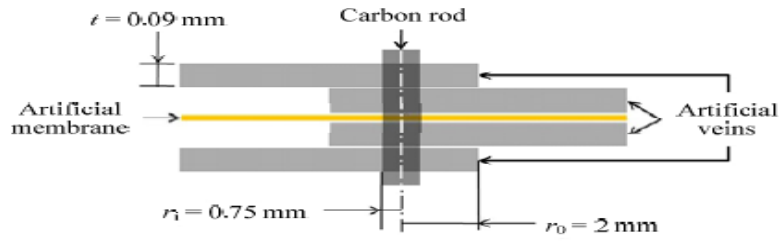

(a) Previous model

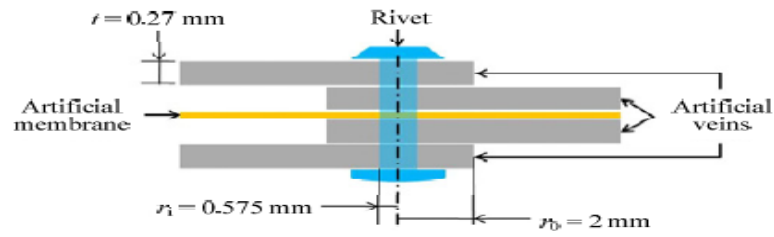

(b) Current mode

Fig.14: Artificial foldable hind wings [6]

\subsubsection{Actuation system}

For the actuation system to fold and unfold the proposed artificial hind wing, here SMA wires are used [6].SMA wire is typically weak and plastically deformable when its temperature is lower than its transformation range. Fig.15a shows the forward action of SMA which is used for unfolding and Fig. 15b shows a heat-treated SMA wire whose original shape is arbitrary bent which is used for folding of the wing. So the two sets of SMA wires used in this work the original SMA wires for wing unfolding, and the heat-treated wire used for wing folding

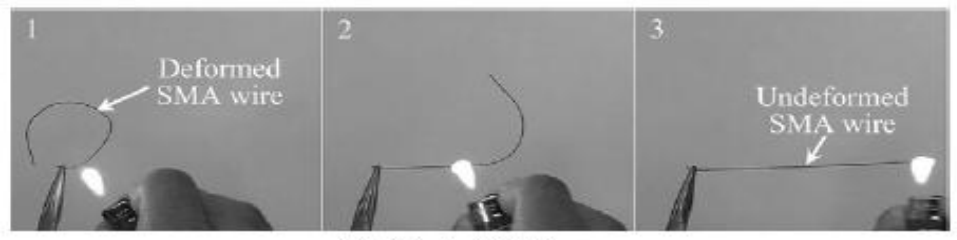

(a) Original SMA wire

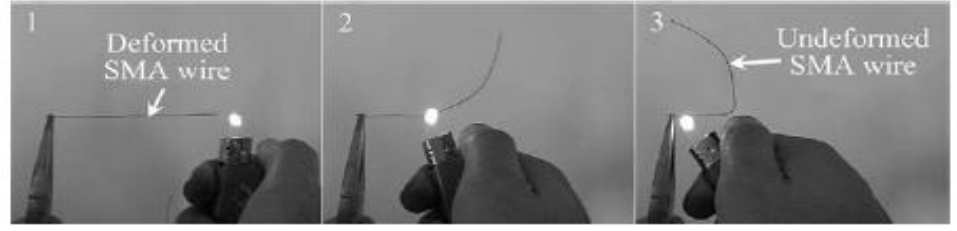

(b) Heat-treated SMA wire

Fig. 15: SMA wire behaviors. [6]

The sequential unfolding and folding motions of the artificial wing are shown in FIG- 16

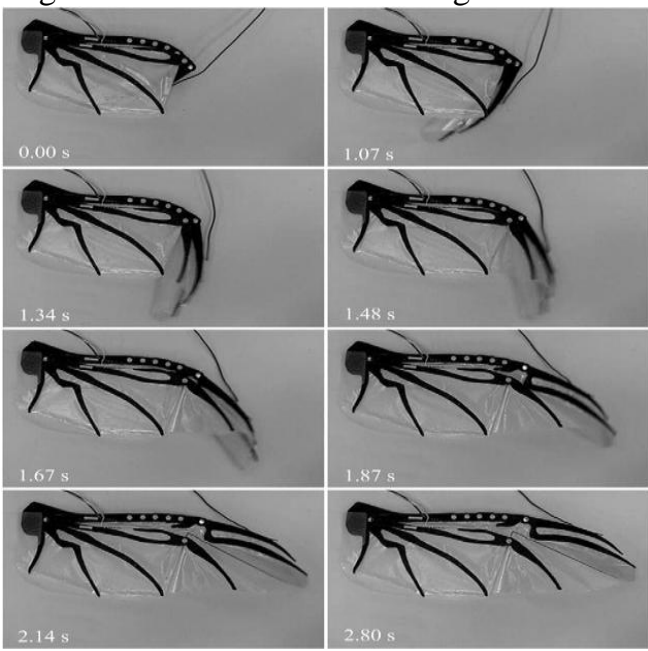

(a) Unfolding 


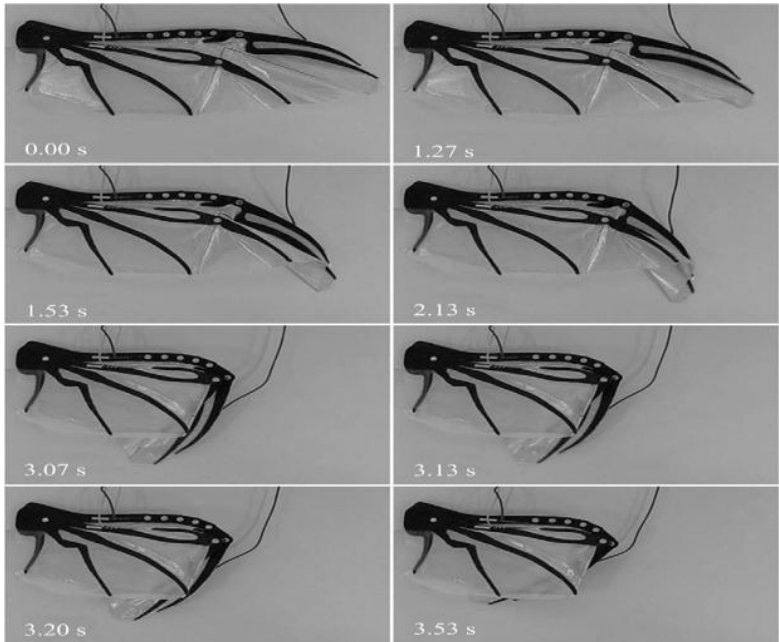

(b) Folding

Fig. 16: Actuation of artificial foldable hind wing [6]

Comparison between real and artificial hind wings [6]

\begin{tabular}{|c|c|c|c|c|c|}
\hline \multirow{3}{*}{ Paramete } & & \multirow{3}{*}{ Real hind wing } & \multicolumn{3}{|c|}{ Artificial hind wing } \\
\hline & & & \multirow{2}{*}{$\begin{array}{c}\text { Previous } \\
\text { (unfolding only) }\end{array}$} & \multicolumn{2}{|c|}{ Present } \\
\hline & & & & Folding wing & Unfolding wing \\
\hline \multicolumn{2}{|l|}{ Length $(\mathrm{cm})$} & 5.2 & 10.5 & \multicolumn{2}{|c|}{10.5} \\
\hline \multicolumn{2}{|l|}{ Weight (g) } & 0.16 & 1.06 & \multicolumn{2}{|c|}{1.1} \\
\hline \multicolumn{2}{|c|}{ Thickness of membrane $(\mu \mathrm{m})$} & $3 \sim 6$ & 15 & \multicolumn{2}{|c|}{15} \\
\hline \multirow{2}{*}{$\begin{array}{l}\text { Typical time } \\
\text { spent (s) }\end{array}$} & Folding & Random & Not available & 4 & Not available \\
\hline & Unfolding & 0.05 & $7 \sim 8$ & Not available & 3 \\
\hline \multirow{2}{*}{$\begin{array}{l}\text { Projected wing } \\
\text { area }\left(\mathrm{cm}^{2}\right)\end{array}$} & Folding & 3.29 & 21.88 & 21.22 & 20.317 \\
\hline & Unfolding & 7.62 & 32.79 & 35.76 & 34.93 \\
\hline \multirow{2}{*}{$\begin{array}{l}\text { Angle between } \\
\text { RA and MP ( }\left(^{\circ}\right)\end{array}$} & Folding & 10 & 12 & 12 & 10 \\
\hline & Unfolding & 20 & 16 & 19 & 19 \\
\hline \multirow{2}{*}{$\begin{array}{l}\text { Angle between RA and } \\
\text { MJ - tip of RA3 (") }\end{array}$} & Folding & 39 & 51 & 50 & 41 \\
\hline & Unfolding & 154 & 148 & 154 & 152 \\
\hline \multicolumn{2}{|l|}{ Folding ratio } & $2.32\left(1.70^{\circ}\right)$ & $1.50^{\circ}$ & $1.70^{\circ}$ & $1.72^{*}$ \\
\hline
\end{tabular}

\section{Conclusions}

\subsection{Conclusions}

This paper discussed a mechanism that generates flapping whose patterns are modeled on dragonflies. In this paper also discussed the improved artificial foldable hind wing by modifying the hinges. Through the modification, the current artificial wing demonstrated swifter folding/unfolding motions, closer angles among veins before and after folding, and a higher folding ratio [6]. In addition, folding motion was discussed by reversing the actuation deformation of the SMA wires through a heat treatment process. Flapping test showed that the artificial hind wing did not fold back during the flapping motion, and possesses an adequate flexibility when flapped at a $9 \mathrm{~Hz}$ wing beat frequency with a $120^{\circ}$ flapping angle.

\subsection{Future work}

Future work will focus on measurement of vertical forces, better wing designs and usage of optimum flapping patterns, control and stabilization. Piezoelectric actuator has the characteristics of high voltage power and small amplitude output when the design is improved by integrating the amplifier mechanism into the flapping wing rotor system, a significant increase of lift would be expected for flapping-wing rotor MAV.

\section{Acknowledgments}

I would like to generously thank my seminar guide, Prof. DNYANESH PAWASKAR for his guidance and constant supervision as well as for providing necessary information regarding the topic. I would also like to thank the library facility of Indian Institute of Technology, Bombay for the literature and journal papers that was required to complete this work. I would also like to thank all those who directly or indirectly helped me in the course of my seminar. 


\section{References}

[1] Hui Hu1 and Anand Gopa Kumar- An Experimental Study of Flexible Membrane Wings in Flapping Flight - Iowa State University, Ames, Iowa, 50011, Gregg Abate3, Air Force Research Laboratory, Elgin Air Force Base, Florida.

[2] http://en.wikipedia.org/wiki/Micro_air_vehicle

[3] helly A. Migita - Effects of low Reynolds numbers on the aerodynamics of micro air vehicles - Department of Mechanical Engineering University of Hawaii at Manoa Honolulu, HI 96822.

[4] Michael A.A. Fenelon, Tomonari Furukawa - Design of an active flapping wing mechanism and a micro aerial vehicle using a rotary actuator - Institute for Robotics and Intelligent Systems (Centre for Artificial Intelligence and Robotics), DRDO, Bangalore 560093, India b Department of Mechanical Engineering, Virginia Polytechnic Institute and State University, Blacksburg, VA, USA

[5] Muhammad A, Park H C, Hwang D Y, Byun D, Goo N S - Mimicking unfolding motion of a beetle hind wing - Chinese Science Bulletin, 2009, 54, 2416-2424.

[6] Improvement of Artificial Foldable Wing Models by Mimicking the Unfolding/Folding Mechanism of a Beetle Hind Wing .Azhar Muhammad1,2, Quoc Viet Nguyen1,2, Hoon Cheol Park1,2,4, Do Y. Hwang4, Doyoung Byun2,3,4, Nam Seo Goo1,2,4.

[7] Haas F, Wootton R J - Two basic mechanisms in insect wing folding - In proceedings of the royal society of London , 1996, 263: 16511658 